\title{
Predominant pathogen competition and core microbiota divergence in chronic airway infection
}

\author{
Geraint B Rogers ${ }^{1,2}$, Christopher J van der Gast ${ }^{3}$ and David J Serisier ${ }^{2,4}$ \\ ${ }^{1}$ SAHMRI Infection and Immunity Theme, School of Medicine, Flinders University, Adelaide, South \\ Australia, Australia; ${ }^{2}$ Immunity, Infection, and Inflammation Program, Mater Research Institute, University \\ of Queensland, Translational Research Institute, Woolloongabba, Queensland, Australia; ${ }^{3}$ NERC Centre for \\ Ecology and Hydrology, Wallingford, UK and ${ }^{4}$ Department of Respiratory Medicine, Mater Adult Hospital, \\ South Brisbane, Queensland, Australia
}

\begin{abstract}
Chronic bacterial lung infections associated with non-cystic fibrosis bronchiectasis represent a substantial and growing health-care burden. Where Pseudomonas aeruginosa is the numerically dominant species within these infections, prognosis is significantly worse. However, in many individuals, Haemophilus influenzae predominates, a scenario associated with less severe disease. The mechanisms that determine which pathogen is most abundant are not known. We hypothesised that the distribution of $\boldsymbol{H}$. influenzae and $\boldsymbol{P}$. aeruginosa would be consistent with strong interspecific competition effects. Further, we hypothesised that where $\boldsymbol{P}$. aeruginosa is predominant, it is associated with a distinct 'accessory microbiota' that reflects a significant interaction between this pathogen and the wider bacterial community. To test these hypotheses, we analysed 16S rRNA gene pyrosequencing data generated previously from 60 adult bronchiectasis patients, whose airway microbiota was dominated by either $\boldsymbol{P}$. aeruginosa or $\boldsymbol{H}$. influenzae. The relative abundances of the two dominant species in their respective groups were not significantly different, and when present in the opposite pathogen group the two species were found to be in very low abundance, if at all. These findings are consistent with strong competition effects, moving towards competitive exclusion. Ordination analysis indicated that the distribution of the core microbiota associated with each pathogen, readjusted after removal of the dominant species, was significantly divergent (analysis of similarity (ANOSIM), $R=0.07, P=0.019$ ). Taken together, these findings suggest that both interspecific competition and also direct and/or indirect interactions between the predominant species and the wider bacterial community may contribute to the predominance of $\boldsymbol{P}$. aeruginosa in a subset of bronchiectasis lung infections.
\end{abstract}

The ISME Journal (2015) 9, 217-225; doi:10.1038/ismej.2014.124; published online 18 July 2014

\section{Introduction}

The World Health Organization has reported that the global burden of diseases is shifting from communicable to non-communicable diseases, with chronic conditions such as heart disease, strokes and lung diseases now being the chief causes of morbidity and mortality (Lopez et al., 2006). Among the great challenges in studying the causes and treatment of chronic lung infections, is a consequence of Koch's postulates and the subsequent concept of infection pathogenesis summarised by the expression 'one microbe, one disease' (Nelson et al., 2013). Koch's postulates have shaped our understanding of medical microbiology, as many important microbial diseases conform to them.

Correspondence: GB Rogers, SAHMRI Infection and Immunity Theme, School of Medicine, Flinders University, North Terrace, Adelaide, South Australia SA 5001, Australia.

E-mail: geraint.rogers@sahmri.com

Received 11 March 2014; revised 10 June 2014; accepted 13 June 2014; published online 18 July 2014
However, that orthodoxy is being undermined by a growing recognition that diverse important diseases, including chronic lung infections, have a polymicrobial aetiology. This expanding understanding of chronic polymicrobial infections, originating from studies of cystic fibrosis (CF) airway microbiota, is beginning to be translated to other chronic lower respiratory diseases, including non-CF bronchiectasis (hereafter referred to as bronchiectasis).

Bronchiectasis is a chronic airway disease characterised by abnormal destruction and dilation of the large airways, bronchi and bronchioles (Cohen and Sahn, 1999). It is associated with chronic and frequently purulent expectoration, multiple exacerbations and progressive dyspnoea that can become disabling (Ellis et al., 1981; Cohen and Sahn, 1999; Barker, 2002), and represents a substantial and growing health-care burden. A recent US study demonstrated a marked increased prevalence in older populations varying from 4.2/100 000 adults aged 18-34 years to $271.8 / 100000$ aged $\geqslant 75$ years (Weycker et al., 2005). Bronchiectasis often goes unrecognised or is misdiagnosed as asthma or 
chronic obstructive pulmonary disease, leading to an underestimated prevalence. Despite this, bronchiectasis is associated with substantial socioeconomic cost due to the frequent use of primary and secondary health-care resources. An US epidemiological study of bronchiectasis-associated hospitalizations from 1993 to 2006 demonstrated an average annual hospitalization rate of $16.5 / 100000$ population with a significant annual increase of $2.4 \%$ in men and $3 \%$ in women (Seitz et al., 2010), with the cost of managing bronchiectasis appearing to be rising (Joish et al., 2013).

Airway inflammation resulting from chronic bacterial infection is thought to be a significant contributory factor driving disease progression in bronchiectasis (Barker, 2002). The perceived importance of bacterial pathogens in airway disease progression is reflected in the use of antibiotics (Serisier and Martin, 2011; Serisier et al., 2013a), both as maintenance therapy and to treat episodes of acute exacerbation. However, our understanding of the mechanisms that underpin relationships between infection by particular bacterial taxa and clinical outcomes is currently poor. This situation undermines the development of rationales for the selection of particular antibiotic treatment regimes (Serisier, 2012) or potentially specific anti-inflammatory therapy (Visser et al., 2012), and achieving better insight into the manner in which treatments achieve beneficial outcomes. Whereas bronchiectasis can result from a variety of recognised aetiologies, it is often considered idiopathic. Recent studies have revealed a substantial and diverse bacterial microbiota (Tunney et al., 2013; Rogers et al., 2013b, 2014; van der Gast et al., 2014), which are typically dominated by either Haemophilus influenzae or Pseudomonas aeruginosa. Perhaps unsurprisingly given its colonisation of the upper airways in healthy individuals, $H$. influenzae is detectable in lower airway secretions from almost all bronchiectasis patients and is commonly the numerically dominant species (Rogers et al., 2013b, 2014). In contrast, $P$. aeruginosa-dominated infections occur in a smaller number of patients (Rogers et al., 2014) but are associated with an accelerated decline in lung function, more frequent pulmonary exacerbations, greater sputum production and a higher requirement for antibiotic therapy (Evans et al., 1996; Ho et al., 1998; Shoemark et al., 2007; Rogers et al., 2014).

A better understanding of the way in which $P$. aeruginosa interacts with the airway environment could provide important mechanistic insights into chronic infection in this patient group and in chronic respiratory infections more widely. Both the physiochemical characteristics of the airway environment and the composition of the pre-existing lung microbiota are likely to influence the likelihood of $P$. aeruginosa infection (Rogers et al., 2013a). Further, where $P$. aeruginosa dominates the infection microbiota, its growth is likely to further affect the composition of airway environment. This impact of colonisation could occur both directly through the metabolomic (Kozlowska et al., 2013) and secretomic (Bergamini et al., 2012) footprint of $P$. aeruginosa, and, in turn, indirectly by stimulating changes in the host immune response (Bergamini et al., 2012) and the activity of other co-colonising species (Bakkal et al., 2010; Tashiro et al., 2013). Whereas the causality in these interactions is difficult to demonstrate, were such relationships to exist, they would result in both an association between $P$. aeruginosa infection and measures of airway disease, and an association between $P$. aeruginosa infection and microbiota composition. The first of these associations has been well documented. However, to our knowledge, there have been no investigations to assess the second.

We hypothesised that (1) the distribution of $H$. influenzae and $P$. aeruginosa in airway samples would be consistent with strong interspecific competition effects; that is, when $H$. influenzae is the dominant species in a bronchiectasis lung infection, the population size of $P$. aeruginosa will be negatively influenced and vice versa when $P$. aeruginosa is dominant. (2) Where $P$. aeruginosa or $H$. influenzae is dominant species in a bronchiectasis lung infection, they are associated with distinct 'accessory microbiota' that reflect a significant interaction between these pathogens and the wider bacterial community. To test these hypotheses, we analysed 16S rRNA gene pyrosequencing data, generated previously, from 60 adult bronchiectasis patients whose airway microbiota was dominated by either $P$. aeruginosa or $H$. influenzae. $H$. influenzaedominated infections were chosen as a comparator group as they had been shown previously not to differ significantly in total bacterial load, dominant taxon relative abundance or prior antibiotic burden (intravenous, oral and combined), with those where P. aeruginosa was dominant (Rogers et al., 2014). Further, despite differences in disease course, these patients did not differ significantly in serum C-reactive protein levels, or sputum interleukin-8 and interleukin-1 $\beta$ levels (Rogers et al., 2014) common markers of systemic and airway inflammation. To limit the potential effect of antibiotic therapy to influence microbiota composition (Serisier, 2013), a sample set was chosen where there had been a 4-week period of clinical stability before collection, with no supplemental antibiotics administered (Serisier et al., 2013b).

\section{Materials and methods}

The analysis performed here was based on $16 \mathrm{~S}$ ribosomal RNA gene pyrosequencing data generated from induced sputum samples from adult patients with bronchiectasis, as part of the BLESS trial (Serisier et al., 2013b). These data are available through the Sequence Read Archive 
(http://www.ncbi.nlm.nih.gov/sra) under the accession number SRP0356. Details of patient recruitment, sample collection, nucleic acid extraction, PCR amplification pyrosequencing and bioinformatics processing have been published previously (Rogers et al., 2013b; Serisier et al., 2013b; Rogers et al., 2014) and details of these processes are provided here as Supplementary Methods.

Of the 96 samples previously analysed, 26 were $P$. aeruginosa-dominated and 34 were $H$. influenzaedominated. It is these 60 samples on which the analysis presented here is based. Patient details for these patients are shown in Table 1. As described previously, the species-level identification of $P$. aeruginosa and $H$. influenzae was confirmed using specific PCR-based assays in all instances (Supplementary Methods), with identification by pyrosequencing treated as presumptive. Where species-level identities were not corroborated by specific assays, identities are presented at the genus level.

Bacterial taxa within each metacommunity were partitioned into core and satellite groups using the Poisson distribution test as previously described (van der Gast et al., 2011; Rogers et al., 2013c). One-way analysis of variance, regression analysis, coefficients of determination $\left(r^{2}\right)$, and residuals and significance
$(P)$ were calculated using the Minitab software (version 16, Minitab, University Park, PA, USA) as described previously (van der Gast et al., 2011; Rogers et al., 2013c). Canonical correspondence analysis, analysis of similarity (ANOSIM) and similarity of percentages analysis were performed using the PAST (Palaeontological Statistics, version 2.17) program available from the University of Oslo website link (http://folk.uio.no/ohammer/ past) run by Øyvind Hammer. Mann-Whitney tests were performed using Prism (version 5.01, GraphPad, La Jolla, CA, USA). Where predominant taxa were removed before analysis, the remaining relative abundance measures were rescaled and expressed as percentages.

\section{Results and discussion}

$P$. aeruginosa and $H$. influenzae share certain similarities, for example, they are both Gram negative, rod-shaped, facultative anaerobic Gammaproteobacteria. However, these common opportunistic pathogens are associated with very different clinical courses when dominant in bronchiectasis lung infections. Here the relative abundances of the two predominant species in their respective groups

Table 1 Clinical, treatment and comorbidity data for patient population

\begin{tabular}{|c|c|c|c|c|}
\hline \multirow{3}{*}{ Gender (male:female) } & \multicolumn{2}{|c|}{ P. aeruginosa } & \multicolumn{2}{|c|}{ H. influenzae } \\
\hline & $6: 20$ & & $16: 18$ & \\
\hline & Range & Mean ( \pm s.d.) & Range & $\operatorname{Mean}( \pm s . d)$. \\
\hline \multicolumn{5}{|l|}{ Clinical measures of disease } \\
\hline FEV1\% * & $30.7-94.2$ & $60.5( \pm 18.1)$ & $37.2-114.7$ & $71.2( \pm 15.1)$ \\
\hline Duration of bronchiectasis (years) & $1-70$ & $45.4( \pm 19.6)$ & $10-65$ & $45.4( \pm 17.5)$ \\
\hline Pulmonary exacerbations in prior 12 months & $2-12$ & $6.3( \pm 3.0)$ & $2-7$ & $3.4( \pm 1.3)$ \\
\hline Leicester cough score & $5.5-20.2$ & $14.8( \pm 3.5)$ & $7.7-19.9$ & $15.6( \pm 2.6)$ \\
\hline SGRQ total & $8.6-79.6$ & $39.9( \pm 16.6)$ & $14.9-58.9$ & $36.7( \pm 14.0)$ \\
\hline Six-minute walk test & $291-650$ & $488.7( \pm 85.1)$ & $275-710$ & $519.6( \pm 101.6)$ \\
\hline C-reactive protein $\left(\mathrm{mg} \mathrm{l}^{-1}\right)$ & $0-21$ & $6.8( \pm 6.0)$ & 0-19 & $7.1( \pm 6.1)$ \\
\hline Induced sputum IL-8 (ng ml-1) & $27.4-1053.8$ & $337.6( \pm 340.3)$ & $28.8-1326.7$ & $275.2( \pm 295.7)$ \\
\hline Induced sputum IL-1 $\beta\left(\mathrm{ng} \mathrm{ml}^{-1}\right)$ & $0.25-36.2$ & $9.8( \pm 10.7)$ & $0.6-115.8$ & $11.4( \pm 22.3)$ \\
\hline \multicolumn{5}{|l|}{ Treatment } \\
\hline Short acting $\beta$-agonist & 14 & & 10 & \\
\hline Inhaled corticosteroid & 16 & & 17 & \\
\hline Inhaled corticosteroid + long acting $\beta$-agonist & 13 & & 10 & \\
\hline Anti-cholinergics & 6 & & 2 & \\
\hline Aspirin & 3 & & 9 & \\
\hline Beta blocker & 0 & & 3 & \\
\hline Nasal steroids & 2 & & 4 & \\
\hline Prednisolone & 3 & & 1 & \\
\hline Antihypertensive & 9 & & 16 & \\
\hline \multicolumn{5}{|l|}{ Comorbidity } \\
\hline Cerebrovascular disease & 1 & & 4 & \\
\hline Heart disease & 2 & & 4 & \\
\hline Hypertension & 6 & & 14 & \\
\hline Diabetes & 1 & & 0 & \\
\hline
\end{tabular}

Abbreviations: $\mathrm{FEV}_{1} \%$ *, forced expiratory volume in 1 s; SGRQ, St George's Respiratory Questionnaire.

$\mathrm{FEV}_{1} \%^{*}$, expressed as a percentage of predicted and measured following administration of a bronchodilator. SGRQ, range 1-100, lower scores indicate better quality of life. Leicester cough score, lower scores indicate worse cough symptoms. 
were high and not significantly different (analysis of variance, $\mathrm{F}_{1,58}=0.096, P=0.758 ; P$. aeruginosa mean abundance and s.d. $=87.3 \pm 13.4 \%$ and H. influenzae $=86.0 \pm 17.9 \%$; Figure 1). When present in the opposite dominated group, the two species were found to be in only very low abundances (P. aeruginosa $=0.37 \pm 1.3 \%$ and $H$. influenzae $=$ $0.56 \pm 0.77 \%$; analysis of variance, $F_{1,58}=0.436$, $P=0.511$ ). Furthermore, $P$. aeruginosa was not detected in 12 from 34 samples of the $H$. influenzae group, and 2 from 26 for $H$. influenzae in the $P$. aeruginosa group (Figure 1). This is consistent with strong competition effects between the two species, moving towards competitive exclusion of the inferior competitor species, and more so for $P$. aeruginosa within the $H$. influenzae group. If these patterns of dominance and suppression could be purely explained by the process of interspecies competition then no between-group differences in accessory microbiota would be expected. However, the analyses performed here demonstrate that taxa present in the microbiota associated with $P$. aeruginosa and $H$. influenzae predominance are significantly divergent.

The distribution of the two sets of microbiota, as determined by direct ordination using Bray-Curtis similarity measures, is shown in Figure 2. Where the dominant taxa ( $P$. aeruginosa or $H$. influenzae) were included (Figure 2a), divergence in the distribution of the microbiota was pronounced and statistically significant (ANOSIM, $R=1, P<0.0001$ ). However, given the high proportion of total bacterial abundance that these predominant taxa would account for within the microbiota (Figure 1), much of the variation between the two groups will result from their inclusion in the analysis. In order to assess whether accessory microbiota composition differed significantly between the two groups, the predominant taxa were removed and the relative abundances of the remaining taxa redistributed and expressed as percentages. When subjected to ordination analysis, the distribution of the accessory microbiota composition (Figure 1b) was not found to be significantly divergent (ANOSM, $R=0.036, P=0.11$ ).

Many accessory microbiota taxa are of low relative abundance. The potential contribution to accessory microbiota of transient bacterial populations within the airways, as opposed to populations of chronically infective bacteria, is therefore high. In order to reduce the effect of these satellite taxa, the core microbiota (composed of non-randomly distributed taxa) in each of the two groups was determined (Figure 3). This is an approach that has been applied successfully in the analysis of chronic bacterial infections associated with CF (van der Gast et al., 2011; Rogers et al., 2013a). In the Haemophilus-dominated group, 9 of the 92 taxa detected were classified as core and 83 as satellite, and in the Pseudomonas-dominated group, 8 of the 70 taxa detected were classified as core and 62 as satellite. In each case, the contribution of individual taxa to the core microbiota was assessed using the similarity of percentages analysis (Table 2 and Supplementary Table S1). Ordination analysis was then performed using the core taxa, and again the difference between the distribution of the core microbiota that included the predominant $P$. aeruginosa and $H$. influenzae populations was significant (ANOSM, $R=1, P<0.0001$; Figure4a). Moreover, the divergence in the distribution of the core microbiota, readjusted after removal of the dominant species, was also found to be significantly divergent (ANOSIM, $R=0.07, P=0.019$; Figure 4b). Satellite taxa were not significantly different between the groups (ANOSIM, $R=0.05, P=0.06$ ).

To assess whether significant differences existed in the relative abundances of specific core taxa between the $P$. aeruginosa- and $H$. influenzaedominated samples, Mann-Whitney tests were used and performed on readjusted core taxa abundance data after $P$. aeruginosa and $H$. Influenzae are removed. This process identified Prevotella spp. and Flavobacterium spp. as being significantly

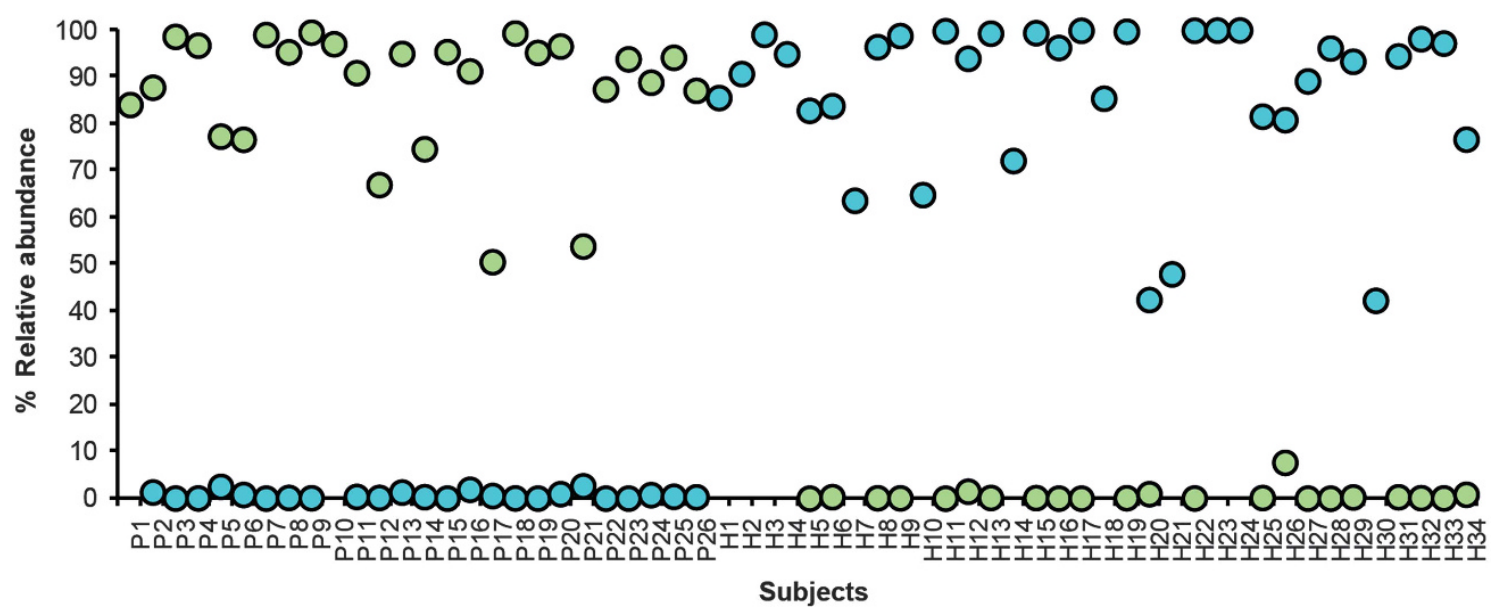

Figure 1 Relative percentage abundances of P. aeruginosa (green circles) and H. influenzae (blue) in samples from within P. aeruginosaand $H$. influenzae-dominated groups (P1-P26 and H1-H34, respectively). 
a

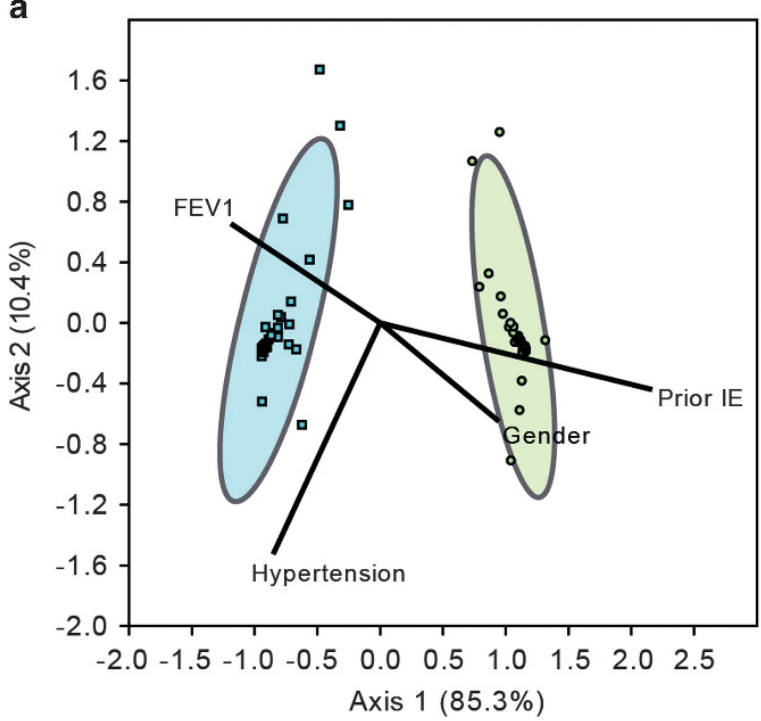

b

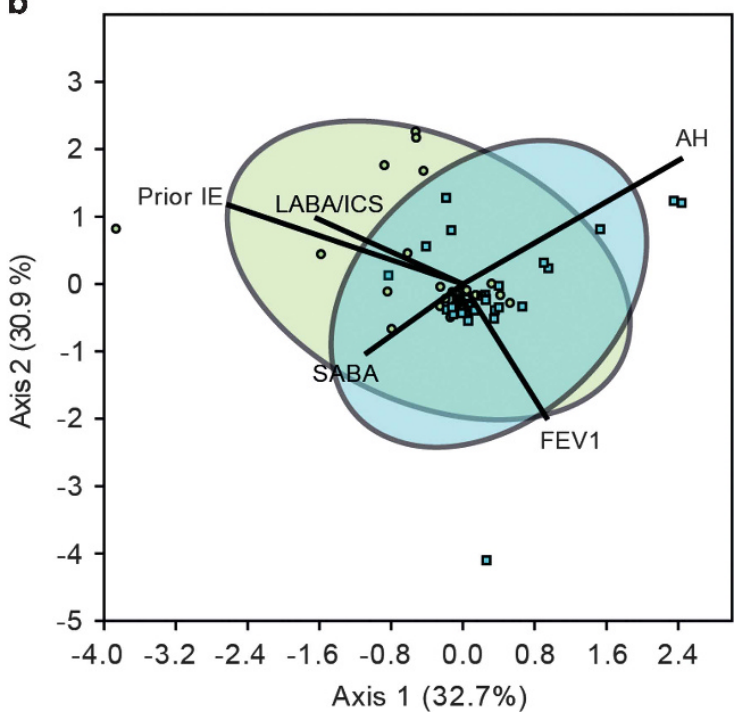

Figure 2 Canonical correspondence biplots for microbiota (a) with and (b) without Pseudomonas and Haemophilus included. Dots represent microbiota samples from the Pseudomonas (denoted with green filled circles) and Haemophilus (blue filled squares) groups. In each instance, the 95\% concentration ellipses are given for the Pseudomonas (green) and Haemophilus (blue) groups. Biplot lines for the clinical variables included in the analyses show the direction of increase for each variable, and the length of each line indicates the degree of correlation with the ordination axes. CCA field labels: 'AH', antihypertensive; 'LABA/ICS', long acting $\beta$-agonist; 'SABA', short acting $\beta$-agonist; 'prior IE', number of pulmonary exacerbations in the prior 12 months. Percentage of community variation explained by each axis is given in parentheses.
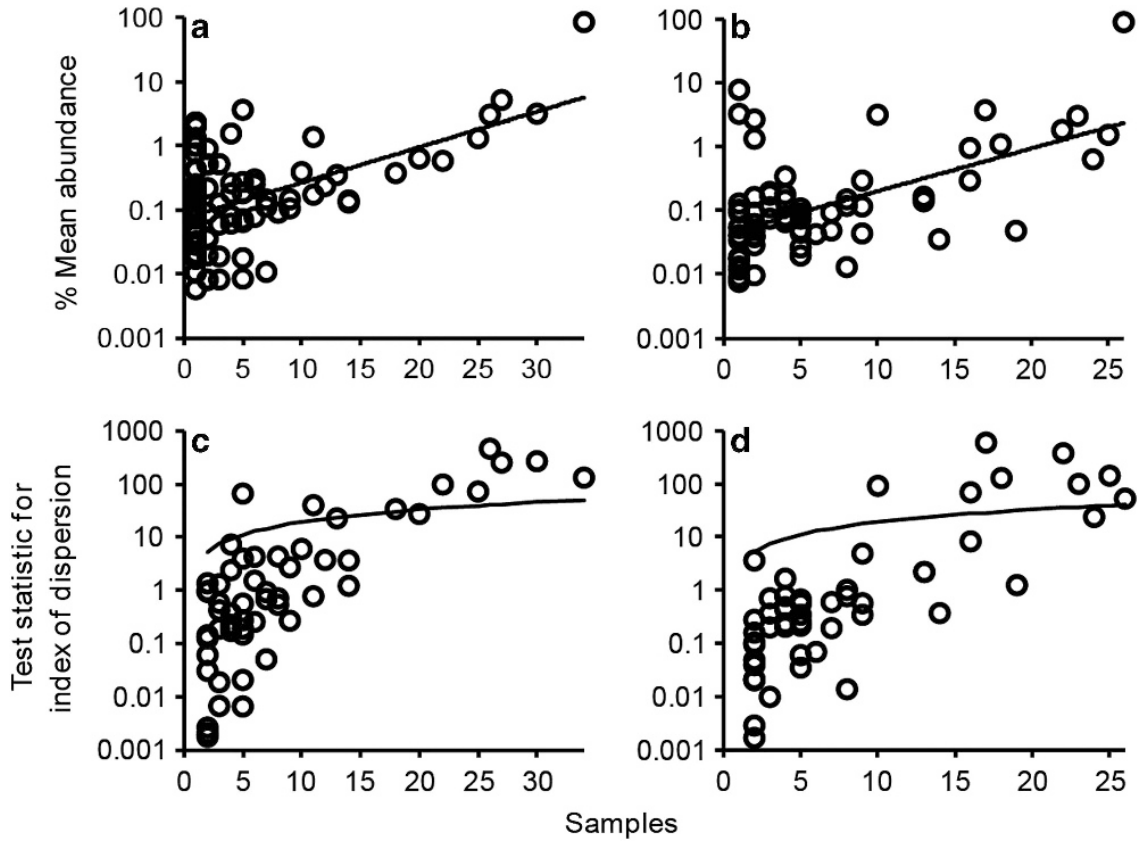

Figure 3 Distribution and dispersal of bacterial taxa among Haemophilus- and Pseudomonas-dominated microbiota samples. (a and b) The number of samples for which each detected bacterial taxon (open circles) was observed, plotted against the abundance (log ${ }_{10}$ scale) of that species among all samples within each group ((a) Haemophilus group, $r^{2}=0.27, \mathrm{~F}_{1.92}=33.2, P<0.0001$; and (b) Pseudomonas group, $\left.r^{2}=0.33, F_{1,68}=33.5, P<0.0001\right)$. In addition, given are dispersal plots to identify which bacterial taxa are randomly distributed within the (c) Haemophilus and (d) Pseudomonas groups; a measure used to assign core versus satellite status. Index of dispersion was calculated as the ratio of variance to the mean of abundance for each taxon within each group and plotted for each sample. The line depicts the $2.5 \%$ confidence limit for the $\chi^{2}$ distribution. Taxa that fall below this line are randomly distributed and were considered satellite taxa, whereas those that are above the line are non-randomly distributed and were considered core taxa. The $97.5 \%$ confidence limit was not plotted, as no taxon fell below that line.

more abundant in the $P$. aeruginosa-dominated samples $(P<0.0001$ and $P=0.003$, respectively), whereas Neisseria spp. was significantly more abundant in $H$. influenzae-dominated samples $(P<0.0001)$. Flavobacterium is a genera that has been reported previously to contribute to bacterial 
Table 2 Similarity of percentages (SIMPER) analysis of bacterial community dissimilarity (Bray-Curtis) between core taxa groups without Pseudomonas and Haemophilus

\begin{tabular}{|c|c|c|c|c|c|c|c|}
\hline \multirow[t]{2}{*}{ Taxon } & \multicolumn{2}{|c|}{$\%$ Mean abundance } & \multicolumn{2}{|c|}{ Samples detected in } & \multirow{2}{*}{$\begin{array}{c}\text { Average } \\
\text { dissimilarity }\end{array}$} & \multirow{2}{*}{$\begin{array}{c}\% \\
\text { Contribution }\end{array}$} & \multirow{2}{*}{$\begin{array}{c}\text { Cumulative } \\
\%\end{array}$} \\
\hline & $\begin{array}{l}\text { Pseudomonas } \\
\text { group }\end{array}$ & $\begin{array}{l}\text { Haemophilus } \\
\text { group }\end{array}$ & $\begin{array}{l}\text { Pseudomonas } \\
\text { group }\end{array}$ & $\begin{array}{l}\text { Haemophilus } \\
\text { group }\end{array}$ & & & \\
\hline Prevotella & 31.9 & 31 & 23 & 27 & 14.96 & 24.17 & 24.17 \\
\hline Veillonella & 20.8 & 26.7 & 25 & 30 & 11.85 & 19.14 & 43.31 \\
\hline Streptococcus & 13.2 & 18.3 & 22 & 26 & 10.51 & 16.99 & 60.30 \\
\hline Moraxella & 14 & 4.9 & 10 & 5 & 8.69 & 14.04 & 74.34 \\
\hline Neisseria & 7.8 & 13.6 & 18 & 25 & 7.59 & 12.26 & 86.60 \\
\hline Flavobacterium & 8.7 & 0 & 17 & 0 & 4.35 & 7.02 & 93.62 \\
\hline Leptotrichia & 3.6 & 2.0 & 16 & 18 & 2.22 & 3.59 & 97.21 \\
\hline Fusobacterium & 0 & 3.5 & 0 & 11 & 1.72 & 2.79 & 100 \\
\hline
\end{tabular}

Given is the mean \% abundance of sequences for each taxon across the samples they were observed to occupy. Analysis was based on an average of 10647 sequences per sample (s.d. 5070, range 2395-28 916). In addition, given is the average dissimilarity between samples (overall mean $=61.9 \%$ ). Percentage contribution is the mean contribution divided by the mean dissimilarity across samples. SIMPER analysis with Pseudomonas and Haemophilus is presented in Supplementary Table S1.

a

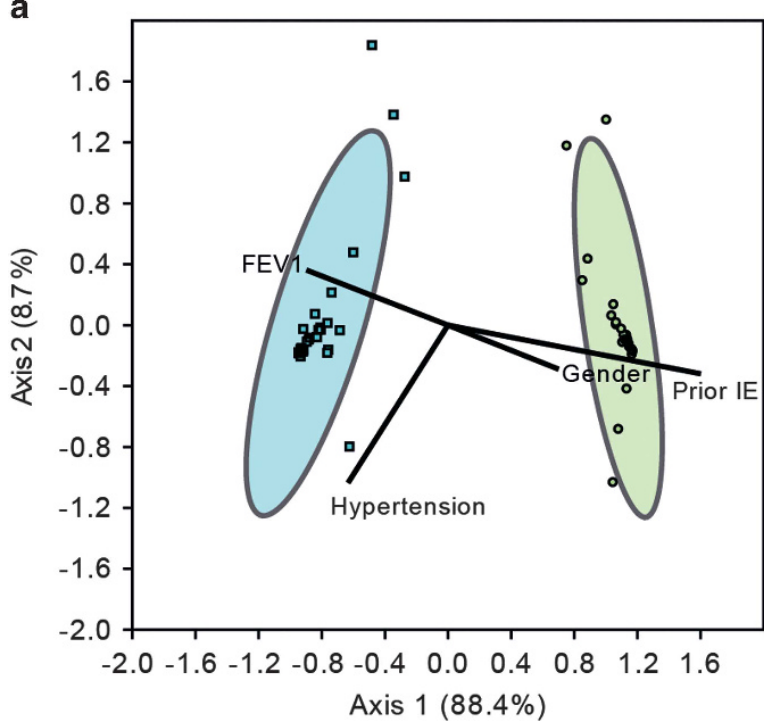

b

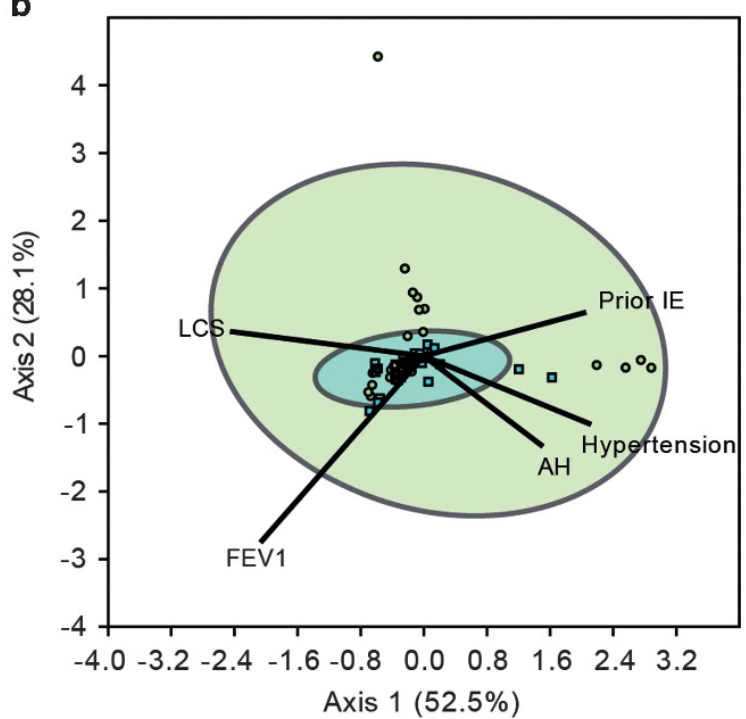

Figure 4 Canonical correspondence biplots for core microbiota (a) with and (b) without Pseudomonas and Haemophilus included. Dots represent core microbiota from the Pseudomonas (denoted with green filled circles) and Haemophilus (blue filled squares) groups. In each instance, the 95\% concentration ellipses are given for the Pseudomonas (green) and Haemophilus (blue) groups. Biplot lines for the clinical variables included in the analyses show the direction of increase for each variable, and the length of each line indicates the degree of correlation with the ordination axes. CCA field labels: 'AH', antihypertensive; 'LCS', Leicester cough score; 'prior IE', number of pulmonary exacerbations in the prior 12 months. Percentage of community variation explained by each axis is given in parentheses.

communities present in chronic lung infections (Rogers et al., 2003, 2004; van der Gast et al., 2011; Rogers et al., 2013a), although typically present at low relative abundances. In contrast, Prevotella spp. have been reported as both common and often at high abundance in both bronchiectasis and CF lung infections (Tunney et al., 2008; Field et al., 2010; Stressmann et al., 2012) and otitis media (Brook, 2008). Prevotella spp. appear to be particularly prevalent when co-colonising with $P$. aeruginosa, a factor that has led to the previous suggestion that a synergistic relationship exists between $P$. aeruginosa and members of this genus (Su and Hassett, 2012). The genus Prevotella is composed of species that are obligate anaerobes. Whereas P. aeruginosa and $H$. influenzae are both capable of fermentation and growth under anaerobic conditions (Schobert and Jahn, 2010; Langereis and Hermans, 2013), the contributions of thick mucoid secretions that $P$. aeruginosa can produce in the airways (Ma et al., 2012), a trait not demonstrated by $H$. influenzae (Langereis and Hermans, 2013), may contribute to reduced oxygen permeation, leading to the creation of greater opportunities for the growth of strict anaerobes, such as Prevotella spp. This model would be consistent with the association observed here between H. influenzae and Neisseria, a genus of typically aerobic species. 
Canonical correspondence analysis was performed next to assess the extent to which variance in the microbiota distribution can be accounted for by variation in measures of disease severity, the presence of comorbidities and non-antibiotic therapies. The results of these analyses are shown in Table 3, and additionally superimposed on Figures 2 and 4. In keeping with previous reports (Rogers et al., 2014), the presence of $P$. aeruginosa in samples as the predominant taxon was associated with high pulmonary exacerbation frequency and poor lung function (low forced expiratory volume in $1 \mathrm{~s}$ percent-predicted), with these factors varying significantly with microbiota distribution. However, whereas such clinical measures are associated with the presence of $P$. aeruginosa, these analyses also show that a significant relationship exists with the wider airway microbiota; a significant relationship was identified here between the variance in core taxa and Leicester Cough Score (a measure of cough symptom severity).

Here we observed distributions of predominant taxa consistent with strong interspecific competition, supporting competitive exclusion in some instances. However, we also observed, for instance, that where $P$. aeruginosa is the numerically dominant species in a bronchiectasis lung infection it is associated with a distinct accessory microbiota; suggesting in addition to interspecific competition, there are also direct and/or indirect interactions between the predominant species and the core microbiota. To some extent, such an effect was also observed for $H$. influenzae but was far less pronounced. However, several different models could explain such interactions. For example, the predominant species could influence the accessory microbiota composition through modification of the airway environment and alteration of its selective properties (and vice versa); here, perhaps the fact that $H$. influenzae is a common resident of the upper airways means that its presence is less disruptive to the commonly occurring infective lower airway microbiota. Alternatively, the same change in the characteristics of the airway could occur through intermediary interaction with the host that results in an altered inflammatory profile, airway secretion composition or secretion clearance rate. Here, a wide array of virulence factors and pro-inflammatory traits possessed by $P$. aeruginosa (Sadikot et al., 2005) may contribute to the magnitude of the effect of its predominance on the accessory airway microbiota. Finally, external influences, such as antibiotic therapy, are likely to contribute to selective pressures in the airway environment. While there was no significant difference in historical antibiotic burden in the $H$. influenzae- and $P$. aeruginosadominated groups, the contribution of more subtle differences in treatment history cannot be excluded. We suggest that no single process is responsible for the associations observed, and rather a dynamic interaction between many different factors give rise to the various types of microbiological scenario seen in vivo. The potential complexity of these interactions makes their elucidation challenging. However, discerning their basis is important, given that a number of important clinical questions arise from the findings we present here. These questions include: (1) could accessory microbiota composition predict subsequent $P$. aeruginosa predominance and its associated poor prognosis? (2) Could intervention aimed at altering the characteristics of the airway environment, or the composition of the accessory microbiota, reduce the likelihood of $P$. aeruginosa infection and predominance? (3) What are the mechanisms to promote the dominance of $H$. influenzae, and as a consequence competitively exclude or suppress $P$. aeruginosa?

In each case, addressing these questions will require in vitro competition experiments between the two dominant bacterial species and members of the core microbiota that we have identified as having likely interactions with $P$. aeruginosa and $H$. influenzae. In addition, longitudinal sample sets that span both clinically and microbiologically important time periods will allow us to better understand the in vivo mechanisms that lead to

Table 3 Canonical correspondence analyses for determination of percent variation in lung microbiota from bronchiectasis subjects by clinical variables

\begin{tabular}{|c|c|c|c|c|c|c|c|c|}
\hline \multirow[t]{2}{*}{ Variable } & \multicolumn{2}{|c|}{ Whole microbiota } & \multicolumn{2}{|c|}{$\begin{array}{l}\text { Whole microbiota without } P \\
\text { and } H\end{array}$} & \multicolumn{2}{|c|}{ Core microbiota } & \multicolumn{2}{|c|}{$\begin{array}{c}\text { Core microbiota without } \\
P \text { and } H\end{array}$} \\
\hline & $\%$ Of variance & Probability & $\%$ Of variance & Probability & $\%$ Of variance & Probability & $\%$ Of variance & Probability \\
\hline Prior exacerbations & 11.35 & 0.01 & 2.35 & 0.02 & 13.8 & 0.01 & 2.7 & 0.01 \\
\hline $\mathrm{FEV}_{1} \%$ predicted & 4.60 & 0.01 & 2.41 & 0.01 & 5.5 & 0.01 & 4.9 & 0.01 \\
\hline Hypertension & 3.08 & 0.01 & - & - & 3.5 & 0.01 & 2.7 & 0.01 \\
\hline Gender & 3.01 & 0.01 & - & - & 3.4 & 0.01 & - & - \\
\hline Antihypertensive & - & - & 2.20 & 0.01 & - & - & 2.3 & 0.01 \\
\hline $\mathrm{LABA}+\mathrm{ICS}$ & - & - & 1.81 & 0.02 & - & - & - & - \\
\hline SABA & - & - & 1.54 & 0.02 & - & - & - & - \\
\hline Leicester cough score & - & - & - & - & - & - & 3.1 & 0.02 \\
\hline
\end{tabular}

Abbreviations: FEV1, forced expiratory volume in $1 \mathrm{~s}$; H, Haemophilus; ICS, inhaled corticosteroid; LABA, long acting $\beta$-agonist; SABA, short acting $\beta$-agonist; P, Pseudomonas. 
predominance of $H$. influenzae or $P$. aeruginosa and its associated worse clinical outcomes. However, obtaining informative longitudinal sample sets in adult bronchiectasis has significant challenges. Intensive, long-term sample collection would be required to span rare and unpredictable events, such as the acquisition of $P$. aeruginosa in a condition that has relatively slow progression (Martínez-García et al., 2007) and is commonly idiopathic (Anwar et al., 2013). Given their potential to provide mechanistic insight into the relationship between recognised airway pathogens, the wider airway microbiota, host immunity and clinical outcome, such long-term frequent sample collection, represent an important next step.

In conclusion, we present evidence supporting the contribution of both interspecific competition and direct and/or indirect interactions between predominant infective taxa and the wider bacterial community, to determine whether $H$. influenzae or $P$. aeruginosa dominates the chronic lung infections associate with bronchiectasis. Given the prognostic implications of $P$. aeruginosa dominance, these findings provide a basis for identifying the mechanisms that underpin this airway microbial ecology, and perhaps offering novel therapeutic opportunities.

\section{Conflict of Interest}

The authors declare no conflict of interest.

\section{Acknowledgements}

This study was supported by the Mater Adult Respiratory Research Trust Fund.

\section{References}

Anwar GA, McDonnell MJ, Worthy SA, Bourke SC, Afolabi G, Lordan J et al. (2013). Phenotyping adults with non-cystic fibrosis bronchiectasis: a prospective observational cohort study. Respir Med 107: 1001-1007.

Bakkal S, Robinson SM, Ordonez CL, Waltz DA, Riley MA. (2010). Role of bacteriocins in mediating interactions of bacterial isolates taken from cystic fibrosis patients. Microbiology 156: 2058-5067.

Barker AF. (2002). Bronchiectasis. $N$ Engl J Med 246: 1383-1393.

Bergamini G, Di Silvestre D, Mauri P, Cigana C, Bragonzi A, De Palma A et al. (2012). MudPIT analysis of released proteins in Pseudomonas aeruginosa laboratory and clinical strains in relation to pro-inflammatory effects. Integr Biol (Camb) 4: 270-279.

Brook I. (2008). The role of anaerobic bacteria in chronic suppurative otitis media in children: implications for medical therapy. Anaerobe 14: 297-300.

Cohen M, Sahn SA. (1999). Bronchiectasis in systematic diseases. Chest 116: 1063-1074.
Ellis DA, Thornley PE, Wightman AJ, Walker M, Chalmers J, Crofton JW. (1981). Present outlook in bronchiectasis: clinical and social study and review of factors influencing prognosis. Thorax 36: 659-664.

Evans SA, Turner SM, Bosch BJ, Hardy CC, Woodhead MA. (1996). Lung function in bronciectasis: the influence of Pseudomonas aeruginosa. Eur Respir J 9: 1601-1604.

Field TR, Sibley CD, Parkins MD, Rabin HR, Surette MG. (2010). The genus Prevotella in cystic fibrosis airways. Anaerobe 16: 337-344.

Ho PL, Chan KN, Ip MS, Lam WK, Ho CS, Yuen KY, Tsang KW. (1998). The effect of Pseudomonas aeruginosa infection on clinical parameters in steady-state bronchiectasis. Chest 114: 1594-1598.

Joish VN, Splisbury-Cantalupo M, Operschall E, Luong B, Boklage S. (2013). Economic burden of non-cystic fibrosis bronchiectasis in the first year after diagnosis from a US health plan perspective. Appl Health Econ Health Policy 3: 299-304.

Kozlowska J, Rivett DW, Vermeer LS, Carroll MP, Bruce KD, Mason AJ, Rogers GB. (2013). A relationship between Pseudomonal growth behaviour and cystic fibrosis patient lung function identified in a metabolomic investigation. Metabolomics 9: 1262-1273.

Langereis JD, Hermans PW. (2013). Novel concepts in nontypeable Haemophilus influenzae biofilm formation. FEMS Microbiol Lett 346: 81-89.

Lopez AD, Mathers CD, Ezzati M, Jamison DT, Murray CJL. (2006). Global Burden of Disease and Risk Factors. Oxford University Press: USA.

Ma L, Wang S, Wang D, Parsek MR, Wozniak DJ. (2012). The roles of biofilm matrix polysaccharide Psl in mucoid Pseudomonas aeruginosa biofilms. FEMS Immunol Med Microbiol 65: 377-380.

Martínez-García MA, Soler-Cataluña JJ, Perpiñá-Tordera M, Román-Sánchez P, Soriano J. (2007). Factors associated with lung function decline in adult patients with stable non-cystic fibrosis bronchiectasis. Chest 132: 1565-1572.

Nelson A, De Soyza A, Perry JD, Sutcliffe IC, Cummings SP. (2013). Polymicrobial challenges to Koch's postulates: ecological lessons from the bacterial vaginosis and cystic fibrosis microbiomes. Innate Immun 18: 774-783.

Rogers GB, Carroll MP, Serisier DJ, Hockey PM, Jones G, Bruce KD. (2004). Characterization of bacterial community diversity in cystic fibrosis lung infections by use of $16 \mathrm{~S}$ ribosomal DNA terminal restriction fragment length polymorphism profiling. $J$ Clin Microbiol 42: 5176-5183.

Rogers GB, Cuthbertson L, Hoffman LR, Wing PAC, Pope C, Hooftman DAP et al. (2013c). Towards unbiased bacterial community analysis in lower respiratory infections. ISME J 7: 697-706.

Rogers GB, Hart CA, Mason JR, Hughes M, Walshaw MJ, Bruce KD. (2003). Bacterial diversity in cases of lung infection in cystic fibrosis patients: 16S ribosomal DNA (rDNA) length heterogeneity PCR and 16S rDNA terminal restriction fragment length polymorphism profiling. J Clin Microbiol 41: 3548-3558.

Rogers GB, Hoffman LR, Carroll MP, Bruce KD. (2013a). Interpreting infective microbiota: the importance of an ecological perspective. Trends Microbiol 21: 271-276.

Rogers GB, van der Gast CJ, Cuthbertson L, Thomson SK, Bruce KD, Martin ML, Serisier DJ. (2013b). Clinical measures of disease in adult non-CF bronchiectasis correlate with airway microbiota composition. Thorax 68: 731-737. 
Rogers GB, Zain NMM, Bruce KD, Burr LD, Chen AC, Rivett DW et al. (2014). A novel microbiota stratification system predicts future exacerbations in bronchiectasis. Ann Am Thorac Soc 11: 496-503.

Sadikot RT, Blackwell TS, Christman JW, Prince AS. (2005). Pathogen-host interactions in Pseudomonas aeruginosa pneumonia. Am J Respir Crit Care Med 171: 1209-1223.

Schobert M, Jahn D. (2010). Anaerobic physiology of Pseudomonas aeruginosa in the cystic fibrosis lung. Int J Med Microbiol 300: 549-556.

Seitz AE, Olivier KN, Steiner CA, Montes de Oca R, Holland SM, Prevots DR. (2010). Trends and burden of bronchiectasis-associated hospitalizations in the United States, 1993-2006. Chest 138: 944-949.

Serisier DJ. (2012). Inhaled antibiotics for lower respiratory tract infection-focus on ciprofloxacin. Drugs Today 48: 339-351.

Serisier DJ. (2013). Risks of population antimicrobial resistance with chronic macrolide use for inflammatory airways dieases. Lancet Respir Med 1: 262-274.

Serisier DJ, Bilton D, De Soyza A, Thompson PJ, Kolbe J, Greville HW et al. (2013a). Inhaled, dual-release liposomal ciprofloxacin in non-cystic fibrosis bronchiectasis (ORBIT-2): a randomised, double-blind, placebo-controlled trial. Thorax 68: 812-817.

Serisier DJ, Martin ML. (2011). Long-term, low-dose erythromycin in bronchiectasis subjects with frequent infective exacerbations. Respir Med 105: 946-949.

Serisier DJ, Martin ML, McGuckin MA, Lourie R, Chen AC, Brain B et al. (2013b). Effect of long-term, low-dose erythromycin on pulmonary exacerbations among patients with non-cystic fibrosis bronchiectasis: the BLESS randomized controlled trial. JAMA 309: 1260-1267.

Shoemark A, Ozerovitch L, Wilson R. (2007). Aetiology in adult patients with bronchiectasis. Respir Med 101: 1163-1170.
Stressmann FA, Rogers GB, van der Gast CJ, Marsh P, Vermeer LS, Carroll MP et al. (2012). Long-term cultivation-independent microbial diversity analysis demonstrates that bacterial communities infecting the adult cystic fibrosis lung show stability and resilience. Thorax 67: 867-873.

Su S, Hassett DJ. (2012). Anaerobic Pseudomonas aeruginosa and other obligately anaerobic bacterial biofilms growing in the thick airway mucus of chronically infected cystic fibrosis patients: an emerging paradigm or 'Old Hat'? Expert Opin Ther Targets 16: 859-873.

Tashiro Y, Yawata Y, Toyofuku M, Uchiyama H, Nomura N. (2013). Interspecies interaction between Pseudomonas aeruginosa and other microorganisms. Microbes Environ 28: 13-24.

Tunney MM, Einarsson GG, Wei L, Drain M, Klem ER, Cardwell C et al. (2013). Lung microbiota and bacterial abundance in patients with bronchiectasis when clinically stable and during exacerbation. Am J Respir Crit Care Med 187: 1118-1126.

Tunney MM, Field TR, Moriarty TF, Patrick S, Doering G, Muhlebach MS et al. (2008). Detection of anaerobic bacteria in high numbers in sputum from patients with cystic fibrosis. Am J Respir Crit Care Med 177: 995-1001.

van der Gast CJ, Cuthbertson L, Rogers GB, Pope C, Marsh RL, Redding GJ et al. (2014). Three clinically distinct chronic pediatric airway infections share a common core microbiota. Ann Am Thorac Soc Online early, Doi:10.1513/AnnalsATS.201312-456OC.

van der Gast CJ, Walker AW, Stressmann FA, Rogers GB, Scott P, Daniels TW et al. (2011). Partitioning core and satellite taxa from within cystic fibrosis lung bacterial communities. ISME J 5: 780-791.

Visser S., Martin ML, Serisier DJ. (2012). Improvements in cystic fibrosis lung disease and airway inflammation associated with etanercept therapy for cystic fibrosis: a case report. Lung 190: 579-581.

Weycker D, Edelsberg J, Oster G, Tino G. (2005). Prevalence and economic burden of bronchiectasis. Clin Pulm Med 12: 205-209.

Supplementary Information accompanies this paper on The ISME Journal website (http://www.nature.com/ismej) 